\title{
IMPLEMENTACIÓN DEL ABP COMO MÉTODO PARA PROMOVER COMPETENCIAS DE COLABORACIÓN EN UN AMBIENTE VIRTUAL (WEBNODE)
}

\section{PBL IMPLEMENTATION AS A METHOD TO PROMOTE COLLABORATION COMPETENCIES IN A VIRTUAL ENVIRONMENT (WEBNODE)}

\author{
Nohemi Rivera Vázquez nohemirv@hotmail.com \\ Universidad Virtual de Tecnológico de Monterrey, México. \\ Adriana María Agudelo Quiroz adrianaagudeloq@gmail.com \\ Institución educativa Gilberto Alzate Avendaño, sede San Isidro, Colombia. \\ Xitlali Manuela Ramos Arcos ramosxitlali@gmail.com \\ Servicios Internacionales de Trasnporte de Puebla, México. \\ Jackeline Camila Vargas Mateus jky14ca@hotmail.com \\ Alcaldía Municipal, Colombia.
}

\section{RESUMEN}

El artículo refiere a un proyecto de investigación educativa basada en evidencia, con la cual se valoró el uso del Aprendizaje Basado en Problemas (ABP) y su impacto en el trabajo colaborativo a través de una webnode. Esta investigación empleó como método la investigación cualitativa, considerándolo como un estudio de caso de tipo instrumental, ya que el fin último fue la valoración de la relación del ABP y el trabajo colaborativo a través de una plataforma virtual. La entrevista semi-estructurada, un cuestionario y la observación virtual son los instrumentos usados para la recolección de datos, que evidencian que, utilizar el método ABP a través de una webnode facilita el desarrollo de competencias de trabajo colaborativo en los procesos de capacitación docente. 
PALABRAS CLAVE: Aprendizaje Basado en Problemas (ABP), trabajo colaborativo, ambiente virtual, tecnología educativa, TIC.

\section{ABSTRACT}

The article refers to a project-based educational research evidence that evaluated the use of Problem Based Learning (PBL) and its impact on collaborative work through a webnode. This investigation used a qualitative research method, considering an instrumental case study, because the ultimate goal was the evaluation of the relationship of PBL and collaborative work through a virtual platform. The semi-structured interview, a questionnaire and virtual observation are the instruments used for data collection, to show that, using the PBL method through a website facilitates the development of collaborative working skills in teacher training processes.

KEYWORDS: Problem Based Learning (PBL), Collaborative work, e-learning, educational technology, Information and communication technologies (ICT).

\section{INTRODUCCIÓN}

El presente trabajo es el resultado de una investigación llevada a cabo a partir de la implementación de una innovación. Una innovación es cualquier acción intencionada que implica la introducción de una novedad en un método, sistema u organización, el cual cambia su estructura y procedimientos y tiene como resultado una mejora en el producto final (Ramírez, 2012).

Para esta innovación se utilizó el método de Aprendizaje Basado en Problemas (ABP) a través de la implementación de una webnode para apoyar la colaboración y comunicación asincrónica entre docentes, ya que esta herramienta dispone de una gran variedad de funciones para la creación de páginas web, sin la necesidad de instalar o configurar ningún tipo de software. De esta manera, es muy fácil colocar una gran cantidad de elementos interactivos, donde el docente podrá consultar y compartir información de calidad. Por lo tanto, el objetivo principal de la innovación implementada fue la mejora de los procesos de capacitación continua docente a través de una comunicación más fluida y la posibilidad de compartir recursos educativos de calidad; finalmente se pretendió que esto a su vez tuviera un impacto positivo en su práctica educativa.

Este proyecto se llevó a cabo metódicamente, documentando las evidencias, con el fin de sustentar con una investigación científica el impacto que la implementación de este recurso tuvo en el desempeño de los docentes. Este proyecto pretendió evaluar los resultados del uso 
del método ABP y su impacto en el trabajo colaborativo a través del uso de la plataforma webnode como medio de innovación para la capacitación docente.

\subsection{Capacitación continua para los docentes en ambientes a distancia}

Los ambientes virtuales y el uso de las Nuevas Tecnologías de Información y Comunicación han ocasionado cambios en los roles, las relaciones, el discurso y el conocimiento de los alumnos e instructores que participan en el proceso de aprendizaje (Hodge y Collins, 2010). Para maximizar la efectividad de un programa de capacitación para docentes se requiere desarrollar materiales que se presenten de manera continua, así como brindarles soporte y monitoreo constante (Bishop, Giles y Bryant, 2005). Un método para ofrecer a los maestros un apoyo y capacitación continua es a través del uso del Internet. La educación a distancia ofrece ventajas únicas, tales como la asincronicidad y el acceso eficiente a la información (Im y Lee, 2003). Este tipo de capacitaciones requieren de una buena coordinación administrativa, académica y tecnológica, retroalimentación oportuna y un buen diseño instruccional (Farías, Pedraza y Lavín, 2013).

\subsection{Aprendizaje colaborativo facilitado en ambientes virtuales}

Lograr aprendizajes colaborativos no es tarea sencilla. Kauchack y Eggen (1998, citado por Ramírez, 2012) mencionan que es tarea del facilitador motivar y apoyar a los alumnos en el desarrollo de las habilidades de trabajo colaborativo: (1) Que los alumnos sientan un objetivo común de grupo; (2) que cada uno de los integrantes se responsabilice; y (3) saber que cada integrante puede aportar un elemento para el éxito del objetivo. El trabajo colaborativo implica los siguientes procesos: Iniciar la comunicación, conducir la conversación, y tener consciencia del ambiente, la actividad y el equipo (Kraut et al., 2002).

El soporte instruccional a través de una página web reduce los costos, da mayor flexibilidad a los maestros, mejora la comunicación y facilita el acceso a la información, lo cual mejora el aprendizaje. Investigaciones demostraron que los maestros que usaban la página web tenían una actitud más positiva hacia el programa y mayores intenciones de continuar, comparados con los que no tenían acceso (Bishop, Giles y Bryant, 2005). En una capacitación a través de una página web se pueden tener discusiones en línea, las cuales permiten que los alumnos alcancen una mayor comprensión conceptual; en este tipo de trabajo colaborativo es fundamental el papel del profesor al brindar retroalimentación oportuna. Se ha demostrado que las discusiones sincrónicas son útiles para promover la interacción social, mientras que las discusiones asincrónicas promueven la comunicación orientada a la tarea, por lo cual deben utilizarse para distintos fines educativos (Im y Lee, 2003).

\subsection{Aprendizaje Activo y método de Aprendizaje Basado en Problemas (ABP)}

La innovación propuesta se implementó a través del método $A B P$, enmarcado dentro del modelo de aprendizaje activo, el cual utiliza la experiencia como base metodológica. Chirino 
(2004) plantea que este modelo utiliza técnicas didácticas vinculadas en forma directa con el aprendizaje colaborativo. Shuell (1986) expone cinco características del aprendizaje activo: (1) el aprendizaje debe ser autodirigido, (2) autorregulado, (3) constructivo, (4) situado en un contexto particular, y (5) social. El método ABP involucra tres pasos: (1) confrontar el problema, (2) realizar un estudio independiente, y (3) regresar al problema estableciendo áreas de oportunidad. Diversos estudios señalan que esta estrategia puede usarse en ambientes presenciales, b-learning así como e-learning y m-learning, lo cual favorece la comunicación sincrónica y asincrónica (Lozano, 2011). Ramírez (2012) afirma que esta metodología facilita la comunicación, así como el desarrollo de habilidades y actitudes que facilitan la transferencia de lo aprendido a diversos contextos.

Además, Armenta, Salinas y Mortera (2013) mencionan que el ABP en modalidad e-learning: (a) propicia interacciones para el aprendizaje colaborativo; (b) fomenta el aprendizaje profundo y autodirigido; y (c) la investigación individual guía al alumno hacia la resolución del problema. Aplicar el método ABP en una actividad académica impartida en línea facilita las interacciones necesarias para el aprendizaje colaborativo y promueve el aprendizaje profundo mediante el razonamiento crítico de una situación problemática y el aprendizaje autodirigido a través del análisis y la investigación individual.

\section{MARCO CONTEXTUAL}

El proyecto se llevó a cabo en una institución educativa de carácter público, sostenida con fondos del estado colombiano y administrada por el municipio de Medellín a través de la Secretaría de Educación, en la cual se ofrece educación desde nivel preescolar hasta el medio superior. Esta es una de cinco sedes satélites, las cuales cuentan, en total, con más de 4000 estudiantes, 136 docentes, 8 directivos y 2 docentes del aula de apoyo.

Uno de sus objetivos actuales es cualificar la calidad en el servicio educativo. Al ser una institución grande y con sedes distantes, requiere de herramientas y estrategias para la formación continua de sus docentes, así como contar con un modelo de comunicación efectivo que facilite el desarrollo e implementación de planes educativos congruentes con su visión y misión. La labor educativa exige una actualización constante, ya que los docentes tienen la responsabilidad de ejercer acciones pedagógicas efectivas y oportunas para responder a las diversas necesidades que presenten los alumnos (Rose, 2008). La educación enfrenta múltiples retos, entre los cuales se encuentra la evolución tecnológica y la transformación en la forma de comunicar y acceder al conocimiento; a lo que se suma la atención a la diversidad educativa. Según Booth y Ainscow (2000) las instituciones deben atender los procesos escolares con enfoques inclusivos, para lo cual se requiere de conocimiento en prevención e identificación de necesidades, así como habilidad para atender a los estudiantes diversos con pedagogías flexibles; es por ello que la implementación de estrategias que le brinden a los docentes conocimiento pedagógico actualizado es una necesidad. 
Es indispensable fomentar el empoderamiento de los docentes dentro de las aulas para dar una atención oportuna, efectiva y eficiente, dando respuesta a las diversas necesidades de los alumnos. Contar con espacios virtuales favorece una asesoría oportuna, facilita la comunicación y permite compartir las ideas, experiencias y resultados de estudios, lo cual impacta en la práctica educativa. El uso de estos recursos parte de considerar los futuros escenarios en la educación y su relación con la tecnología, así como reflexionar sobre su incidencia y las prácticas de formación cotidiana. Entonces, el propósito de este tipo de implementaciones es innovar a partir de relacionar el uso de las tecnologías con los aprendizajes (Moschen, 2008).

\section{METODOLOGÍA}

Se llevó a cabo un muestreo aleatorio estratificado, lo que implica que se dividió a la población en categorías (los docentes de primero representan una categoría, los de segundo otra, y así sucesivamente); y una vez identificados los estratos se sortearon las unidades para escoger a las que representarían a la muestra de cada estrato, teniendo todos las mismas posibilidades de ser elegidos (Rodríguez, Ariza, y López, 2007). Entonces, la muestra consistió en cuatro docentes de nivel preescolar, cuatro de primer grado, cuatro de segundo y cuatro de quinto de primaria.

La población total era de 24 docentes, y la muestra para el presente estudio se tomaron16. La edad promedio de los profesores es de 45 años de los cuales 22 son mujeres y 2 son hombres. 14 de los docentes cursaron un bachiller en pedagogía y 10 no lo hicieron; 22 estudiaron una licenciatura o pregrado en educación y 2 de ellos no; 16 tienen un postgrado y 8 no lo tienen. En cuanto a sus competencias tecnológicas, 10 de ellos tienen un nivel básico en el manejo de tecnologías, 10 un nivel medio, y 4 un nivel avanzado.

Se diseñó un curso para que los profesores trabajaran durante 6 días a través de la metodología $A B P$, utilizando los foros asincrónicos de comunicación. Al final compartieron su propuesta en el blog de la página para que pudiera ser consultado por los demás equipos y para colocar comentarios. Al finalizar el curso, se aplicó un cuestionario estructurado que constó de dos componentes: el primero evaluaba si se lograron los objetivos principales del curso ABP; y el segundo medía la competencia de trabajo colaborativo conseguida a través del curso ABP y de la interacción con la tecnología novedosa. Dos días después de que se terminó el curso se les realizó una entrevista semiestructurada con preguntas abiertas a través del webnode. La entrevista también estaba dividida en dos componentes para evaluar las variables establecidas, la cual permitió que los participantes expresaran más a detalle sus opiniones y percepciones.. Finalmente, se analizaron las tareas que se colocaron en el blog de la página web. Después se procedió al análisis de los resultados, los cuales fueron entregados después de dos semanas tiempo que duró el análisis- a la institución con el fin de que les sirvieran de base para mejorar la innovación implementada, adaptándose a las necesidades de sus usuarios.

Para el diseño del curso de capacitación a distancia se usó el modelo de aprendizaje activo, a través de su metodología ABP y utilizando la técnica de aprendizaje colaborativo. Los recursos 
educativos consistieron en: lecturas sugeridas y obligatorias, videos y conexión con otros sitios web de consulta. La participación en el curso se llevó a cabo en un ambiente de aprendizaje virtual a través de la interfaz, el correo, los foros de discusión asincrónica y el blog para interactuar con el equipo.

Se empleó un método de investigación cualitativo, definido por Valenzuela y Flores (2012) como un método de indagación cuya meta es comprender a profundidad, describir fenómenos y construir realidades. La indagación fue holística, con el objetivo de estructurar la información en un todo coherente y lógico para describir la naturaleza del fenómeno, su estructura dinámica y sus manifestaciones (Martínez, 2006). Se consideró que el estudio de casos instrumental era el método más adecuado. De acuerdo con Madera y Monasterio (s/f) el estudio de casos se basa en el entendimiento de una situación a través de su análisis y descripción detallada. En este caso se utiliza el estudio de casos instrumental, ya que el fin último no es conocer el contexto escolar particular a profundidad, sino utilizar este contexto como medio para conocer el método ABP y sus efectos en las competencias de colaboración, es decir, el caso sirve como instrumento para conseguir otros fines indagatorios.

Para la colección de datos se elaboraron dos instrumentos: (1) Una entrevista semiestructurada; y (2) un cuestionario estructurado en las que las preguntas y respuestas están predeterminadas (Valenzuela y Flores, 2012). El modo de circulación del cuestionario fue auto-administrado ya que brinda eficacia para la recolección de datos, la organización y el análisis (Giroux \& Tremblay, 2004), se realizaron a través de la misma página web. Además, se observaron las aportaciones en el foro y las presentaciones del blog para dar validez a la información colectada. Los instrumentos descritos anteriormente miden los dos constructos; el ABP como método de enseñanza y el desarrollo de competencias de colaboración, por lo que fueron divididos en las siguientes categorías: (1) Experiencia de implementación del ABP, para la cual se mide si el docente es capaz tanto de identificar y formular la situación problemática, como de proponer alternativas (Fernández, 2008; Armenta, 2003); y (2) las competencias de colaboración usando el webnode, el cual permite medir las acciones colaborativas que se llevaron a cabo en un ambiente virtual. El trabajo colaborativo se midió a través de la presencia social, la participación activa y la colaboración observadas en el ambiente de aprendizaje virtual (Alavi, 1994). Asimismo, se evaluó la usabilidad de la página web, a través del grado de satisfacción y motivación del usuario al estar en contacto con una plataforma interesante, útil, amigable y atractiva (Zambrano, 2007; Alarcón et al., 2013).

Para el análisis de datos se utilizó el método de comparación constante (MCC), el cual consiste en una comparación continua de los hechos con las propiedades de las categorías identificadas, con el fin de encontrar relaciones entre ellos y patrones de comportamiento (Osses, Sánchez e Ibáñez, 2006). Para dar validez y confiabilidad a los resultados se llevó a cabo una triangulación instrumental, la cual consiste en separar los resultados que arrojan dos instrumentos distintos de recolección de datos que pretenden medir las mismas variables (Ramírez, 2013). 


\section{RESULTADOS ALCANZADOS}

Con respecto al método $A B P$, la mayoría de los docentes estuvieron de acuerdo en que el problema fue sencillo de identificar, y que el hecho de resolverlo en equipo les permitió conocer y analizar el tema a profundidad. Asimismo, todos los docentes estuvieron totalmente de acuerdo con que las soluciones a las que se llegó en equipo son factibles de implementarse en su contexto particular. En la entrevista semiestructurada, los docentes opinaron que el problema planteado en el curso facilitó el trabajo colaborativo. Algunos docentes comentaron que el planteamiento del problema los motivó a solicitar las opiniones de otros compañeros, y a escucharlos. Otros afirmaron que el problema tenía una relación directa con la puesta en práctica del conocimiento; finalmente, otro dijo que buscar solución al problema facilitó que se generara una discusión alrededor de un tema de interés común en la que todos podían aportar, sugerir y crear conocimiento, ideas y estrategias.

Los participantes estuvieron de acuerdo en que los cursos de capacitación continua que utilizan esta metodología les ayudaban a ampliar ideas de vanguardia en el campo educativo. En general opinaron que la metodología les permitió tener contacto con docentes de diversas áreas, compartir documentos con sus colegas, mantenerse actualizados y crear grupos de estudio colaborativo con docentes de su institución en incluso de otras partes del mundo que tuvieran intereses en común. Además, la mayor parte de los docentes entrevistados mencionaron que las actividades realizadas bajo el método ABP les ayudaron a mejorar sus habilidades laborales, buscando estrategias, actividades y materiales para la solución de problemas reales. Afirmaron que el curso los motivó a trabajar en favor de sus alumnos, así como a salir de su aislamiento y aprender de las experiencias de sus compañeros.

Con respecto al trabajo colaborativo, ninguno de los docentes manifiesta haber tenido un problema para saber con quién dirigirse para aclarar dudas o buscar asesoría. Confirmaron haber participado activamente en el foro de discusión y haber usado el blog para intercambiar información con sus colegas. Además afirmaron que los comentarios de sus compañeros en el foro les sirvieron para reflexionar, enriquecer y mejorar sus aportaciones, y que en general, el trabajo en equipo contribuyó con la mejora de la comprensión de los temas.

Los docentes comentaron que antes la página tenía un enfoque administrativo y solamente se utilizaba para apoyarlos en su planeación. Sin embargo, les parece que los foros y el blog pueden utilizarse como un medio para solicitar y brindar apoyo entre los docentes porque todos tienen acceso a la plataforma y pueden compartir de manera sincrónica y asincrónica sus necesidades, intereses, materiales, sugerencias y dudas de su trabajo académico. También afirmaron que el trabajo en equipos virtuales ha comenzado a mejorar su ambiente laboral, principalmente porque trabajar a distancia les permite hacerlo de forma asincrónica respetando los tiempos y espacios de cada quién. De la misma manera, todos comentaron que el trabajo en equipos virtuales promueve una comunicación asertiva, porque se trabaja con un objetivo en mente desde un principio y se aprende a respetar las opiniones e ideas de los demás. Además formar equipos de trabajo facilita compartir dudas y proponer alternativas para solucionar sus 
problemas. Finalmente, sugirieron que se continúen implementando cursos de capacitación con esta metodología, donde se aborden temas variados y que en un futuro exista un moderador que esté al pendiente de las discusiones para proporcionar una retroalimentación oportuna. También agradecieron el haber tenido la oportunidad de trabajar colaborativamente.

\section{DISCUSIÓN DE RESULTADOS}

EI ABP ayuda a identificar las necesidades de aprendizaje, profundizar en un tema y llegar a soluciones aplicables en su contexto. Los docentes mostraron una buena disposición ante la actividad, identificaron fácilmente el problema, y reconocieron la relación directa con su práctica. Asimismo afirmaron que el curso había ayudado a mejorar sus habilidades y ampliar sus conocimientos e ideas de vanguardia en el campo educativo, incluso compartieron su intención de utilizar el ABP con sus alumnos, esto apoya los hallazgos de Ramírez (2012) acerca de que el ABP facilita la transferencia de lo aprendido a diversos contextos. Este método fomenta un aprendizaje profundo, por lo tanto, es recomendable implementar cursos que contemplen el método ABP para que los docentes comprendan los temas, y apliquen el conocimiento en su práctica (Armenta, Salinas y Mortera, 2013).

El método ABP facilita el aprendizaje autodirigido, el trabajo colaborativo y la reflexión. Los participantes afirmaron que se sintieron cómodos trabajando a su propio ritmo, los comentarios de sus compañeros les ayudaron a reflexionar sobre el tema, así como a enriquecer sus aportaciones. También manifestaron que el trabajo ABP facilita la comunicación, ya que fomenta el respeto a las opiniones de los demás. Esto respalda lo que expresan Ramírez (2012) y Lozano (2011) con respecto a que el ABP puede usarse en ambientes a distancia, facilitando la comunicación sincrónica y asincrónica. Armenta, Salinas y Mortera (2013) plantean que propicia interacciones para el aprendizaje autodirigido y colaborativo, a través del análisis individual y en equipo. Esto lleva a considerar la metodología ABP de utilidad, si se pretende impulsar a los docentes a que compartan sus experiencias y se apoyen entre sí, sobre todo si se cuenta con la tecnología necesaria para que la colaboración pueda realizarse de manera asincrónica.

El uso de una webnode favorece el intercambio de información y el trabajo colaborativo, lo cual facilita el aprendizaje significativo. Lo expresado por los docentes es que los foros en la plataforma puede usarse para compartir sus necesidades, intereses, materiales, sugerencias, dudas y podrían ser útiles para brindarse apoyo, respetando los tiempos y espacios de cada quien. Bishop, Giles y Bryant (2005) sostienen que la instrucción a través de una página web, como la webnode, brinda flexibilidad a los maestros, mejora la comunicación y facilita el acceso a la información. Es por esto que los centros educativos deben contar con la tecnología necesaria para fomentar un ambiente de trabajo colaborativo, así como invertir en la alfabetización digital de sus docentes. 
Es necesario contar con un facilitador que esté al pendiente de las discusiones que se generen. La necesidad de un moderador que guíe la metodología ABP fue una de las sugerencias proporcionada por los docentes. Esto mismo sostienen Im y Lee (2003) cuando afirman que en estos ambientes es fundamental el papel del profesor al brindar retroalimentación oportuna. Por lo cual se sugiere que cuando se implementa un curso ABP se cuente con el apoyo de uno o varios facilitadores expertos en el tema, que puedan resolver las dudas y brindar retroalimentación a los participantes.

Para que una capacitación con el método ABP sea efectiva es necesario que se efectúe constantemente. La mayoría de los docentes expresaron su deseo de continuar participando en cursos similares. De acuerdo con Bishop, Giles y Bryant (2005), para maximizar la efectividad de una capacitación se requiere desarrollar materiales que se presenten de manera continua, así como brindar soporte y monitoreo constante. No basta con una sola capacitación ABP para notar una mejora en el desempeño, se debe mantener un esfuerzo a través del tiempo para poder observar los beneficios.

\section{CONCLUSIONES}

En un mundo caracterizado por el cambio, se requiere de la implementación oportuna de innovaciones en todos los ámbitos; este cambio educativo requiere de un amplio conocimiento teórico y de la integración de modelos y estrategias de enseñanza congruentes para llevarlo a la práctica promoviendo una filosofía unificada y aprendizaje significativo. La evolución del ser humano ha transformado los procesos de enseñanza-aprendizaje creando nuevas formas culturales, de comunicación, de difusión y acceso a la información que conciben las tecnologías como una herramienta que ofrece valor agregado al proceso educativo. Por lo anterior se considera que el uso del método ABP a través de una webnode fortalece las competencias de trabajo colaborativo; posibilitando un aprendizaje significativo para una mayor transferencia de sus conocimientos y habilidades a la práctica docente, así como un mejor manejo de las tecnologías educativas, en particular el webnode.

Es posible concluir que utilizar el método ABP a través de una página web facilita el desarrollo de competencias de trabajo colaborativo en los procesos de capacitación docente; con esta información se corroboran los descubrimientos de Armenta, Salinas y Mortera (2013) acerca de que el ABP propicia interacciones para el aprendizaje colaborativo. La educación continua se facilita con el uso de tecnologías como medio para incrementar el trabajo colaborativo. Este tipo de soporte mejora la comunicación y proporciona un acceso más eficiente a la información, lo cual implica una mejora en el aprendizaje (Bishop, Giles y Bryant, 2005). Por el otro lado, si dicha tecnología se percibe por el usuario como fácil de usar (usabilidad) se aumenta la satisfacción y motivación de los aprendices a emplearla para su aprendizaje. 
Los constructos abordados en el estudio son temas innovadores y de relevancia para cualquier contexto educativo. Sin embargo, por cuestiones de tiempo y presupuesto, fueron evaluados solamente en una institución, lo cual representa una limitante para la generalización y transferencia de los resultados del proyecto a otros contextos. De la misma manera, debido a que se contó con muy poco tiempo para el desarrollo de la página web, no fue posible aprovechar al máximo el potencial de los medios virtuales en la construcción de conocimiento a través del método ABP. Esta limitación causó que la formación continua a distancia para los docentes no pudiera sustituir completamente la riqueza de la interacción que ocurre en los encuentros presenciales. En futuras investigaciones se utilizarán recursos multimedia y medios de comunicación sincrónica para mejorar la experiencia virtual.

Debido a la limitante mencionada del tiempo, solamente se pudo evaluar una sesión de capacitación. Sin embargo, las competencias relacionadas con el trabajo colaborativo, deben evaluarse de forma continua; el trabajo colaborativo debe ser parte de la identidad en el diseño de las distintas actividades (Ramírez, 2012). Para futuras investigaciones se recomienda llevar a cabo un estudio longitudinal para poder observar cómo evoluciona el trabajo colaborativo después de varias capacitaciones con el método ABP. Otro de los aspectos a considerar es que para algunos de los docentes participantes, el método ABP utilizado de manera virtual, era algo nuevo, por lo cual su desenvolvimiento frente al trabajo no fue tan fluido.

En este trabajo se aborda el uso de la página web solamente para la capacitación continua. Sin embargo, para el desarrollo del trabajo colaborativo es fundamental utilizar estas herramientas tecnológicas como un espacio para que los docentes compartan su quehacer diario, y que sus experiencias queden registradas para la comunidad académica en general. Se recomienda que en futuras investigaciones se mida el impacto que tiene el uso de la tecnología no sólo como medio para brindar capacitación, sino como un espacio para la interacción y el intercambio de información, en especial, en contextos donde el uso de la tecnología no es una generalidad.

Los resultados se validaron con un tamaño de muestra relativamente pequeño. Se espera que en futuras investigaciones se usen muestras más representativas de la población. Además, sería conveniente utilizar un método mixto que ofrece la oportunidad de registrar y evidenciar de manera cuantificada datos que enriquezcan el conocimiento que se tiene acerca del impacto y efectos producidos a partir de la implementación del método ABP para el desarrollo de habilidades de colaboración.

\section{REFERENCIAS}

AINSCOW, M. (2001). Necesidades especiales en el aula, guía para la formación del profesorado. España: Narcea. 
ALARCÓN, P., ÁlVAREZ, X., HERNÁNDEZ, D. y MALDONADO, D. (2013). Matriz de habilidades TIC para el aprendizaje. Santiago de Chile: Ministerio de educación. Recuperado de: http://www.eduteka.org/habilidadestic.php

ALAVI, M. (1994). Computer-mediated collaborative learning: An empirical evaluation. MIS quarterly, 8(2), 159-174.

ARMENTA, D., SALINAS, V. y MORTERA, F. (2013). Aplicación de la técnica educativa aprendizaje basado en problemas para capacitación a distancia. Revista lberoamericana de Educación a Distancia, 16(1), 57-83. Recuperado de: http://ried.utpl.edu.ec/sites/default/files/file/archivo/volumen\%2016_1/Aplicaciontec nicaeducativa.pdf.

BISHOP, D. C., GILES, S. M., \& BRYANT, K. S. (2005). Teacher receptiveness toward web-based training and support. Teaching and Teacher education, 21(1), 3-14.

BOOTH, T. y AINSCOW, M. (2000). Índice de inclusión, Desarrollando el aprendizaje y la participación en las escuelas. Reino Unido: CSIE.

CHIRINO, V. (2012). Aprendizaje activo como estrategia educativa. Documento de trabajo. Tecnológico de Monterrey. México.

FARÍAS, G., PEDRAZA, N. y LAVÍN, J. (2013). Gestión de un programa de capacitación en línea para el desarrollo de habilidades y capacidades TICs en profesores de negocios. Revista Electrónica de Investigación Educativa, 15(1), 45-61. Recuperado de http://redie.uabc.mx/vol15no1/contenido-fariaspedraza.html

FERNÁNDEZ, N. (2008). Gestión de la educación continua en las IES en México. Identificación de los indicadores de desempeño. IV Congreso internacional AMECyD. Universidad Nacional Autónoma de México. Recuperado de: http://www.econtinua.com/documentos/indicadores2008.pdf.

GIROUX, S. y TREMBLAY, G. (2004). Metodología de las ciencias humanas. La investigación en acción. México, D. F: Fondo de Cultura Económica

HODGE, E. \& COLLINS, S. (2010). Collaborative Efforts: Teaching and Learning in Virtual Worlds. EDUCASE Review, 45(3), 62-63. Recuperado de http://www.educause.edu/ero/article/collaborative-efforts-teaching-and-learningvirtual-worlds.

IM, Y., \& LEE, O. (2003). Pedagogical Implications of Online Discussion for Preservice Teacher Training. Journal Of Research On Technology In Education, 36(2), 155-170. 
KRAUT, R. E., FUSSELL, S. R., BRENNAN, S. E. \& SIEGEL, J. (2002). Understanding effects of proximity on collaboration: Implications for technologies to support remote collaborative work. En P. J. Hinds y S. Kiesler, Distributed work (pp. 137-162). Massachusetts, USA: Massachusetts Institute of Technology.

LOZANO, R. A. y HERRERA B. J. A. (2011). Diseño de programas educativos basados en competencias, Monterrey, México: Editorial Digital del Instituto Tecnológico de Monterrey.

MADERA, A. y MONASTERIO, I. (s/f) Estudio de Casos. Universidad Autónoma de Madrid.

MARTÍNEZ, M. (2006). La investigación cualitativa. Revista De Investigación En Psicología, 9(1). Recuperado http://miscursos.itesm.mx/courses/1/UV.ED4027L.1313.1/db/ 1419488 1/INV\%20CU ALITATIVA.pdf.

MOSCHEN, J. (2008). Innovación Educativa. Buenos Aires, Argentina: Bonum.

OSSES, S., SÁNCHEZ, I., e IBÁÑEZ, F. M. (2006). Investigación cualitativa en educación: Hacia la generación de teoría a través del proceso analítico. Estudios pedagógicos, 32(1), 119133.

RAMíREZ, M. S. (2012). Modelos y Estrategias de Enseñanza, Monterrey, México: Editorial Digital del Tecnológico de Monterrey.

RAMíREZ, M. S. (2013). Triangulación de instrumentos para el análisis de resultados, [video]. Disponible en la Escuela de Graduados en Educación de la Universidad Virtual del Tecnológico de Monterrey, en el sitio Web: http://apps05.ruv.itesm.mx/portal/uvtv/video/video.jsp?folio=4626

RODRígueZ, R. P., ARIZA, M. B., y LÓPEZ, F. S. Z. (2007). Abordaje hermenéutico de la investigación cualitativa. Teorías, procesos, técnicas. Bogotá, Colombia: Universidad Cooperativa de Colombia.

ROSE, D. (2008). Guía para el diseño universal del aprendizaje. EEUU: Editorial Cast.

SHUELL, T. J. (1986). Cognitive conceptions of learning. Review of Educational Research, 56, 411436.

VALENZUELA, J. y FLORES M. (2012). Fundamentos de investigación educativa. Monterrey, México: Editorial Digital del Tecnológico de Monterrey. 
ZAMBRANO, F. (2007). La usabilidad, entre la tecnología y la pedagogía. Revista Digital Universitaria, $8(5)$. Recuperado

de http://www.revista.unam.mx/vol.8/num5/art35/int35.htm.

Rivera, N.; Agudelo, A.; Ramos, X. M.; \& Vargas, J. C. (2015). Implementación del ABP como método para promover competencias de colaboración en un ambiente virtual (webnode). EDUTEC, Revista Electrónica de Tecnología Educativa, 51. Recuperado el dd/mm/aa de http://www.edutec.es/revista 